\title{
Splenic immune responses during canine visceral leishmaniasis
}

\author{
Dalit STRAUSS-AYALI ${ }^{\mathrm{a}, \mathrm{b}, \mathrm{c} *}$, Gad BANETH $^{\mathrm{a}}$, Charles L. JAFFE ${ }^{\mathrm{c}}$ \\ ${ }^{\text {a }}$ School of Veterinary Medicine, Hebrew University of Jerusalem, PO Box 12, Rehovot 76100, Israel \\ ${ }^{\mathrm{b}}$ Present address: Department of Cell Biology and Molecular Genetics, 1105 Microbiology Bldg., \\ University of Maryland, College Park, MD 20742, USA \\ ${ }^{\mathrm{c}}$ The Kuvin Center for the Study of Tropical and Infectious Diseases, Hebrew University - Hadassah \\ Medical School, Jerusalem, Israel
}

(Received 4 October 2006; accepted 9 January 2007)

\begin{abstract}
Dogs are the main reservoir host for zoonotic visceral leishmaniasis caused by Leishmania infantum. In this study we investigated the immune response in spleens of $L$. infantum-infected dogs by measuring the mRNA expression levels for a wide panel of cytokines, transcription factors and chemokines. mRNA levels and parasite load were followed during 7 months of experimental infection and 14 months post-treatment, and were compared to naturally-infected (NI) dogs. Similarly, serum anti-Leishmania IgG and IgG subclass levels were measured during experimental infection. An increase in IFN- $\gamma$, T-bet, IP-10 and RANTES was found in the experimentally and NI dogs, implicating a substantial type-1 immune response during canine visceral leishmaniasis. IL-4, a type-2 associated cytokine, increased as early as one month after experimental infection, while IL-5 was high at later stages. Interestingly, the expression levels of the Treg-associated cytokines, IL-10 and TGF- $\beta$, did not change during the infection. Total anti-Leishmania IgG and IgG subclasses increased during the experimental infection. However, no association with specific cytokine patterns was observed. Parasite load in the spleens increased as early as one month postinfection and remained high until treatment. The load was higher in the polysymptomatic NI dogs than in the experimentally-infected dogs. This study indicates that both type- 1 and type- 2 immune responses occur in the spleen during canine $L$. infantum infection, and suggests that the early elevation of IL-4 might have a role in the persistence of parasites in the presence of high IFN- $\gamma$ expression.
\end{abstract}

canine visceral leishmaniasis / IFN- $\gamma$ / IL-4 / T-bet / IP-10

\section{INTRODUCTION}

Zoonotic visceral leishmaniasis (ZVL) is caused by parasites belonging to the Leishmania donovani complex (L. d. infantum syn. $L$. $d$. chagasi) and is widespread in the Mediterranean Basin, Middle East and South America. Dogs are the main reservoir host for this parasite in endemic

*Corresponding author: strauss@umd.edu areas. Following transmission, these parasites multiply in the skin at the infection site and in the absence of an efficient protective immune response they migrate to the viscera and establish an infection in the spleen, liver and bone marrow. This eventually results in a symptomatic and fatal disease unless treated. Interruption of parasite transmission during canine visceral leishmaniasis (CVL) was shown to 
reduce disease incidence in humans [18]. However, current treatment regimens for CVL are not fully effective for eradicating these parasites [28]. A commercial vaccine against CVL [5], is available in parts of South America but not in the Old World.

The murine Leishmania major model of leishmaniasis was the first model where the $\mathrm{T}$ helper 1 and 2 dichotomy was studied and shown to be associated with resistance and susceptibility to an infectious disease [37]. It is now widely accepted that protective immunity against Leishmania parasites is mediated by type-1 immune responses [26]. However, infection of $L$. major-susceptible inbred mouse strains with the visceralizing parasite, $L$. donovani, manifested a mixed pattern of Th1 and Th2 cytokines resulting in resistance [27]. Moreover, researchers were unable to clearly associate a Th2 polarity with non-healing, reactivating, or systemic leishmaniasis in humans. Interestingly, the severity of human visceral leishmaniasis (HVL) has not been associated with increased levels of IL-4 but of IL-10, which was also detected in the spleen of infected patients [23]. Similarly, elevated levels of IFN- $\gamma$ were found in biopsies taken from the spleen, lymph nodes and bone marrow of infected people $[19,21,23]$, indicating that an unfavorable disease prognosis in humans is not related to a defect in Th1 cell response but rather to a concurrent expression of the cytokine IL-10. Interleukin-10, traditionally thought to be produced mainly by macrophages, is secreted by naturally occurring CD4+CD25+ $\mathrm{T}$ regulatory (Treg) cells in lesions of low dose L. major infected C57BL/6 mice. This cytokine appears to be responsible for the suppression of Leishmania-specific Th1 immunity and prevention of sterile cure in this model [3].

Understanding the cellular immune responses of dogs to infection with Leishmania parasites is crucial for vaccine design. It will also be instrumental in discover- ing efficient treatment modalities that can decrease the prevalence of ZVL. Nevertheless, only little information is currently available on these responses in vivo. Involvement of IL-10 in the immune response of Leishmania-infected dogs has not yet been established, since the expression of IL-10 mRNA in antigen stimulated PBMC or the bone marrow of infected dogs was not elevated during the course of an experimental infection, or in naturallyinfected dogs, respectively [31,36]. Expression of IL-4 mRNA was found in the bone marrow of some symptomatic dogs [31], and significantly higher IL-4 expression was recently detected locally in skin lesions from $L$. infantum-infected dogs [7]. Interferon- $\gamma$ expression of antigen stimulated PBMC, in skin lesions and in the bone marrow of infected dogs was shown to be elevated [7, 10,31,41].

The critical role of chemokines and their receptors in the development of immunity against a wide variety of pathogens has become a focus of interest in recent years $[9,42]$. Chemokines are often differentially induced in response to various stimuli or pathogens as a reflection of their varied ability to activate $\mathrm{T}$ cells and macrophages, influence $T$ cell differentiation and recruit appropriate effector cells to the site of inflammation. The involvement of chemokines in leishmaniasis was investigated primarily using murine models of leishmaniasis [13, 35, 44]. A few studies have examined chemokine responses locally in the skin or peripheral blood mononuclear cells of people with leishmaniasis $[20,34]$. However, no information is available on the pattern of chemokine expression in visceral organs during HVL or during CVL.

The aim of this study was to characterize the components of the spleen immune response in dogs, a natural host of visceral leishmaniasis, during disease development and treatment, and to evaluate the association between immune 
responses and parasitic load. The expression levels of various cytokines and transcription factors associated with type-1 (IFN- $\gamma$, TNF- $\alpha$, IL-12, IL-18 and T-bet), and with type- 2 immune responses (IL-4, IL-5 and GATA-3), and with an inhibition of the immune response associated with Treg cells (IL-10, TGF- $\beta$ ) were evaluated using a quantitative real-time PCR. In addition, several chemokines (IP-10, RANTES, MIP-1 $\alpha$, MCP-1) that have been associated with the secretion of distinct type- 1 and type- 2 cytokines [1,22], macrophage activation $[8,15]$, and with the recruitment of specific $\mathrm{T}$ cell populations $[16,33]$ were analyzed. Using an experimental model of CVL we followed the kinetics of spleen cytokine, transcription factor and chemokine expression levels during 7 months of an oligosymptomatic infection and then for 14 more months after treatment. These levels were compared to expression levels in dogs with polysymptomatic natural CVL, and correlated to parasite load in the spleen.

\section{MATERIALS AND METHODS}

\subsection{Animals}

The first group of dogs consisted of six male beagles ( 6 months old, Wt $($ mean $\pm \mathrm{SE}) 12.58 \pm 0.36 \mathrm{~kg}$ ) that were studied following an experimental infection [40,41]. In brief, the dogs were infected intravenously with $8.6 \times 10^{8}$ L. infantum amastigotes. Two laboratorybred $L$. infantum free male beagle dogs (6 months old, $12.25 \pm 0.17 \mathrm{~kg}$ ) served as uninfected controls (second group). Experimentally-infected (EI) dogs and their controls were monitored for 7 months post-infection. EI dogs were then treated for three months with oral allopurinol (20 mg/Kg B.W./day), and their response to therapy was followed for 14 additional months (24 months altogether). The absence of $L$. infantum parasites was verified in EI and control dogs prior to initiation of this study by evaluating cultures and PCR of spleen and lymph node aspirates, and by conjunctival PCR [40]. The dogs were negative for $L$. infantum on all tests. The third group consisted of 10 naturallyinfected (NI) mixed breed dogs (Age (mean): 5.35 years, 5 males and 5 females); 6 were evaluated for chemokine expression and 10 for cytokine and transcription factor expression. The NI dogs were seropositive for anti-Leishmania IgG when evaluated by ELISA (mean OD = 1.488).

All L. infantum EI dogs were classified as oligosymptomatic according to the semi-quantitative scoring method suggested by Quinnell et al. [31]. These dogs $(n=6)$ demonstrated relatively mild skin involvement manifested as hair loss and fine exfoliation of the ear tips (pineal alopecia) (5/6). Other clinical signs typical of CVL included enlargement of the lymph nodes and/or spleen (6/6), and conjunctivitis (1/6). All clinical signs resolved in response to treatment. Uninfected control dogs did not exhibit any clinical signs typical of CVL. The albumin to globulin ratio in the blood of EI dogs, decreased below normal values by 5 and 7 months post-infection (median $\mathrm{A} / \mathrm{G}$ ratio $=0.59$ and 0.45 , respectively; Suppl. Fig. $1 \mathrm{~A}^{1}$ ). These values were also lower than the control dogs at 3,5, and 7 months post-infection, as well as immediately after treatment $(p<0.05)$. Similar decreases were measured for white blood cells (WBC) counts $(p<0.05 ; 3,5$, and 7 months post-infection), hematocrit and platelet counts ( $p<0.05 ; 3$ and 7 months post-infection) (Suppl. Fig. $1 \mathrm{~B}-\mathrm{D}^{1}$, respectively). Biochemical parameters used to evaluate kidney function (urea and creatinine) were all within the normal range except for one dog that showed elevated serum urea and creatinine beginning

\footnotetext{
${ }^{1}$ Supplementary data are available online only at www.vetres.org.
} 
2 months after the treatment was stopped. Renal disease is a common finding in CVL and usually results from immune-mediated glomerulonephritis. After the treatment period the dog did not show any external clinical signs that were compatible with leishmaniasis. However, it was humanely euthanized 3 months before the end of the study because of chronic kidney disease. All $(n=10)$ L. infantum naturallyinfected dogs exhibited multiple typical clinical signs of leishmaniasis (lymphadenomegaly (10/10), splenomegaly (9/10), alopecia $(8 / 10)$, dermatitis $(8 / 10)$, ocular lesions (4/10), onychogryposis (2/10) and bleeding tendencies $(1 / 10))$. According to the same semi-quantitative score, all the NI dogs were classified as polysymptomatic. The albumin to globulin ratio in the blood of these NI dogs was below the normal $\left(\right.$ median A/G ratio $=0.35$, Suppl. Tab. $\mathrm{I}^{1}$ ), as were their hematocrit and platelet values (Suppl. Tab. $\mathrm{I}^{1}$ ). While 2 of the NI dogs had elevated kidney parameters, the median urea and creatinine values for this group were within the normal range (Suppl. Tab. $\mathrm{I}^{1}$ ).

After clinical evaluation, dogs were anesthetized with $0.075 \mathrm{mg} / \mathrm{kg} \quad$ B.W of acepromazine s.c. followed by i.v. administration of ketamine hydrochloride $(10 \mathrm{mg} / \mathrm{kg} \quad$ B.W $)$ and diazepam (1 $\mathrm{mg} / \mathrm{kg} \mathrm{B.W}$ ). Fine needle aspirates were taken from the spleen of EI and control dogs during the infection and from EI dogs after the treatment was stopped (Suppl. Fig. 2 ${ }^{1}$ ). Spleen aspirates were similarly obtained from the NI dogs at the time of diagnosis. Aspirates were snapped frozen in liquid nitrogen, when intended for later RNA extraction, or kept on ice when intended for later DNA extractions, and were then stored at $-70{ }^{\circ} \mathrm{C}$. Serum was obtained from the EI dogs for longitudinal measurement of anti-Leishmania total IgG and IgG subclasses (Suppl. Fig. $2^{1}$ ). This study was approved by the Animal Ethics Committee of the Hebrew
University of Jerusalem (MD-64.18-06; DHHS animal welfare assurance number OPRR-A01-5011).

\subsection{Leishmania infantum real-time PCR}

Quantification of the Leishmania parasite load in the spleen was carried out using a Leishmania qPCR previously described by Wortmann et al. [43], that was adapted for use with a canine system in this study. The target for the canine qPCR was the dog single copy gene: prostaglandin $\mathrm{E}_{2}$ receptor subtype EP2 $\left(P G E_{2} R\right)$ gene (GenBank accession number AF075602). The primers (365F;

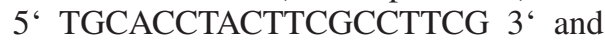

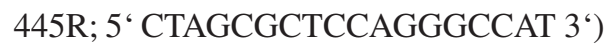
were designed ("Primer Express" software, Applied Biosystems, Foster City, CA, USA) to amplify an $80 \mathrm{bp}$ fragment, and used with a flurogenic probe (5 ${ }^{\circ} \mathrm{FAM}$ ATGACCTTCTTCAGCCTGGCCACG 3 TAMRA). Amplifications were performed in duplicate using the Geneamp 5700 system (Applied Biosystems) in a $25 \mu \mathrm{L}$ reaction mix containing $2.5 \mu \mathrm{L}$ DNA, $900 \mathrm{nM}$ of each primer, $200 \mathrm{nM}$ fluorogenic probe, $5.5 \mathrm{mM} \mathrm{MgCl} 2,200 \mu \mathrm{M}$ of each dNTP, $0.025 \mathrm{U} / \mu \mathrm{L}$ Hot Goldstar Taq polymerase and $10 \times$ buffer (qPCR Core Kit - Eurogentec, Seraing, Belgium). The sensitivity of the Leishmania TaqMan system was evaluated using DNA extracted from 10-fold dilutions of $L$. infantum stationary phase promastigotes (MCAN/IL/2001/LRC-L1020) in a fixed number of WBC obtained from a healthy Leishmania negative dog. Using these standards, this assay was able to detect down to 0.25 parasites per sample. The sensitivity of the canine PCR system was evaluated using ten fold dilutions of DNA extracted from WBC of a healthy Leishmania negative dog. This system which amplified the $P G E_{2} R$ single copy gene was able to detect as little as 25 WBC per 
sample. No parasites were detected with DNA obtained from healthy CVL negative canine WBC. Similarly, parasite DNA was not amplified using the canine system. Both TaqMan systems, as well as the appropriate non-template controls, reagent controls and standards were always run on the same plate using DNA obtained from canine splenic aspirates. The number of parasites and canine cells was obtained for each sample, based on the standard curves. The number of canine cells was then adjusted to a fixed number of canine cells $\left(10^{6}\right)$, and the number of parasites in the same samples was multiplied by the same factor, allowing comparison between the samples. The number obtained represented the number of parasites per $10^{6}$ canine cells and was designated as the PL (parasite load).

\subsection{Cytokine, transcription factor and chemokine quantitative PCR assays}

Total RNA was extracted from spleen aspirates using the RNAeasy mini kit (Qiagen $\mathrm{GmbH}$, Hilden, Germany). RNA extraction, as well as further RT-PCR and real-time PCR, were done in batches containing samples from one dog at various time points (when aspirates were taken from EI and control dogs). Extracted RNA was then treated with RQ1 RNAse free DNAse (Promega, Madison, WI, USA) according to the manufacturer's protocol. Aliquots of DNAse treated RNA were kept at $-70{ }^{\circ} \mathrm{C}$ until subjected to RT-PCR using the RT-iT kit (Abgene, Epsom, UK). Briefly, each RNA sample (200 ng) was added to $1 \mu \mathrm{L}$ of Oligo dT and adjusted to $12 \mu \mathrm{L}$ using RNAse free $\mathrm{ddH}_{2} \mathrm{O}$. After 5 min of heating to $70{ }^{\circ} \mathrm{C}$, RNA was cooled and an $8 \mu \mathrm{L}$ mixture consisting of $5 \times 1$ st strand buffer $(4 \mu \mathrm{L}), 5 \mathrm{mM}$ dNTP mix $(2 \mu \mathrm{L}), 0.1 \mathrm{mM}$ DTT $(1 \mu \mathrm{L})$, and RTiT mix $(1 \mu \mathrm{L})$, was added to each sample.
Samples were further heated to $47^{\circ} \mathrm{C}$ for $40 \mathrm{~min}$, followed by heating to $75^{\circ} \mathrm{C}$ for $10 \mathrm{~min}$, and cooling to $4^{\circ} \mathrm{C}$. cDNA samples were then kept at $-20^{\circ} \mathrm{C}$ until use. To control for residual genomic DNA contamination, a non-RT sample was obtained at one sampling point for each dog by adding to the RNA the oligo-dT, $\mathrm{ddH}_{2} \mathrm{O}, 5 \times 1 \mathrm{st}$ strand buffer, and DTT only.

Quantification of canine cytokine, transcription factor and chemokine transcripts was carried out by real-time PCR, using the Geneamp 5700 system (Applied Biosystems) with the DNA binding dye SYBR Green I (qPCR Core Kit for SYBR Green I, Eurogentec, Seraing, Belgium). The canine $\beta$-actin gene was used as a reference. Reverse and forward primer sequences were established using the Primer Express software (Applied Biosystems) (Suppl. Tab. $\mathrm{II}^{1}$ ) based on published canine sequences (IFN- $\gamma$, TNF- $\alpha$, IL-4, IL-5, IL-10, TGF- $\beta$, IP-10, RANTES, MIP- $1 \alpha$, MCP-1 and $\beta$-actin), or deduced from areas of high homology in other species when known canine sequences were not available (T-bet and GATA-3). Primers for IL-18 and IL-12p40 were previously published by Peters et al. [30]. Cultured DH82 cells (malignant canine histiocyte cell line, kindly obtained from Dr Shimon Harrus) were used as a source of cDNA for qPCR optimization (either stimulated with LPS (Sigma Aldrich, Rehovot, Israel): $\beta$-actin, IL-18, TGF- $\beta$, TNF- $\alpha$, IL-10, RANTES, or not stimulated with LPS: MIP- $1 \alpha$ and MCP-1). cDNA obtained from Concanavalin A (Sigma Aldrich) stimulated peripheral blood mononuclear cells from a healthy dog was used for the optimization of IFN- $\gamma$, IL-4, IL-5, IL12p40, T-bet, GATA-3 and IP-10 qPCR, together with non-RT and non-template controls. Final primer and $\mathrm{MgCl}_{2}$ concentrations are detailed in Suppl. Table $\mathrm{II}^{1}$. PCR products were sequenced to verify their homology to the relevant mRNA sequences. The sequence of T-bet 
amplification product had $100 \%$ homology to a predicted canine T-bet mRNA sequence (GenBank XM 548164) and to a sequence on chromosome 9 of the dog (13748146-13748246; GenBank NW 139866.1). The sequence for the GATA-3 amplification product had $100 \%$ homology to a sequence on chromosome 2 of the dog (21493-20598; GenBank AAEX 01006157.1).

Due to a lack of RNA template with sufficient gene copies the reaction efficiencies were analyzed by running 10-fold dilutions of cleaned PCR product as was previously published by Peters et al. [29]. Since these were in the range of $95-105 \%$ it enabled the use of a relative quantitation method expressed as the $2^{\Delta \mathrm{Ct}}$, also previously described by Peters et al. [30].

\subsection{Total IgG and IgG subclass ELISA}

The presence of anti-Leishmania total IgG was measured in EI and NI dog sera using an ELISA, as previously described. The cut-off value was set at 0.6OD [2]. $\mathrm{IgG}$ subclasses were measured in sera obtained from the L. infantum - EI dogs before infection, at 2, 4, 6 and 8 weeks, 3,5 and 7 months post-infection, and at 7 months after treatment was stopped (Suppl. Fig. $2^{1}$ ). IgG subclass ELISA were performed using a panel of mAbs [25] (kindly provided by M.J. Day), and the protocol previously described by Quinnell et al. [32] with minor changes. Crude antigen $(30 \mu \mathrm{g} / \mathrm{mL})$ prepared from $L$. infantum promastigotes (MCAN/IL/1994/LRCL369) was used for coating the plates. Serum obtained from each dog was tested using all IgG subclass mAbs on one ELISA plate. Known positive and negative controls were run for each subclass on every plate, thus enabling standardization between the different runs.

\subsection{Statistical analysis}

Differences between infected and uninfected dogs were evaluated using the Wilcoxon rank sum test, while differences between EI dogs at different time points were analyzed using the Wilcoxon signed rank test. Correlations were done using the Spearman rank correlation test. Analysis was done using the Statistix software (Analytical Software, Tallahassee, FL, USA).

\section{RESULTS}

\subsection{Spleen parasite load}

All EI dogs had detectable Leishmania DNA in their spleens as early as one month after the experimental infection (Tab. I). Parasite load decreased and remained relatively constant at 3 and 5 months postinfection (Tab. I), but dropped even further at 7 months when compared to 1 month post-infection. Immediately after treatment parasite load decreased dramatically and remained very low until the end of the study 14 months later (Tab. I). Evidence of parasite DNA was observed in a random fashion for 3 or 4 of the treated dogs at each sampling point. L. infantum NI dogs had a higher parasite load when compared to EI dogs at 3 and 7 months post-infection (Tab. I).

\subsection{Cytokine, transcription factor and chemokine expression levels in the spleen during the experimental infection}

Among the type- 1 cytokines that were evaluated in this study, the expression levels of IFN- $\gamma$ increased most prominently in the spleen following infection (Fig. 1). More specifically, IFN $-\gamma$ expression was elevated in infected dogs compared to their pre-infection values, at 3 and 5 months 


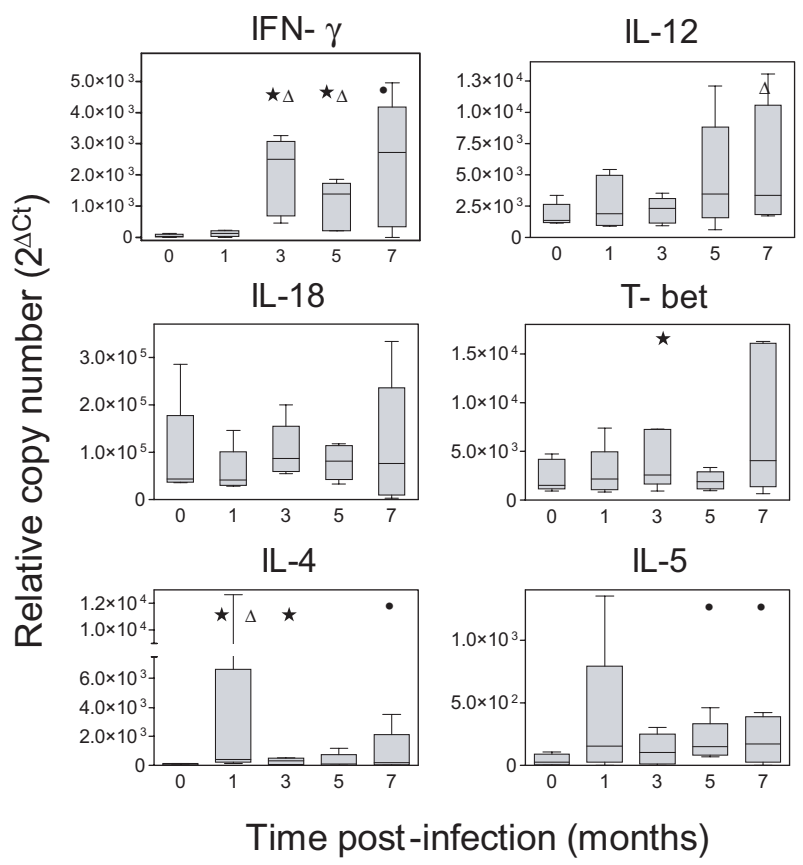

Figure 1. Cytokine and transcription factor gene expression during the course of experimental $L$. infantum infection in dogs. Spleen mRNA expression of IFN- $\gamma$, IL-12, IL-18, T-bet, IL-4 and IL-5, analyzed using SYBR green qPCR in EI dogs $(n=6)$. Box plots show the medians (horizontal lines across the box), interquartile ranges (vertical ends of the box), and whiskers (lines extending from the box to the highest and lowest values). Values that are different from pre-infection values $\left(p<0.05^{\star}, p<0.063 \bullet\right)$ are indicated, as well as those different from uninfected control dogs $(n=2 ; p<0.05 \Delta)$.

Table I. Parasite load in the spleen of Leishmania infantum experimentally and naturally-infected dogs.

\begin{tabular}{lccccccccc}
\hline & & & $\begin{array}{c}\text { Experimental } \\
\text { infection }\end{array}$ & & & & $\begin{array}{c}\text { Post } \\
\text { treatment }^{\mathrm{a}}\end{array}$ & $\begin{array}{c}\text { Natural } \\
\text { infection }\end{array}$ \\
\hline Time (months) & 0 & 1 & 3 & 5 & 7 & 0 & 7 & 14 & \\
\hline $\begin{array}{l}\text { Median parasite } \\
\text { load (PL) }\end{array}$ & $0^{\mathrm{b}}$ & 592.14 & 244.43 & 375.11 & $200.88^{\mathrm{c}}$ & $0.015^{\mathrm{d}}$ & $0.1^{\mathrm{d}}$ & $0.07^{\mathrm{d}}$ & $1714.16^{\mathrm{e}}$ \\
$\begin{array}{l}25 \text { th-75th } \\
\text { percentiles }\end{array}$ & $(0-0)$ & $(205-1072)$ & $(136-1010.2)$ & $(265-1019)$ & $(26-493)$ & $(0-1.37)$ & $(0-11.35)$ & $(0-6.9)$ & $(434.4-5602)$ \\
\hline
\end{tabular}

\footnotetext{
a Parasite load following treatment of experimentally-infected dogs.

b Values are median number of parasites standardized to $10^{6}$ canine cells.

${ }^{c}$ Different from the PL value of 1 month post-infection $(p<0.05)$.

${ }^{\mathrm{d}}$ Different from the PL value at 7 months post-infection $(p<0.05)$.

${ }^{\mathrm{e}}$ Different from the experimentally-infected dogs PL values of 3 and 7 months post-infection $(p<0.05)$.
} 


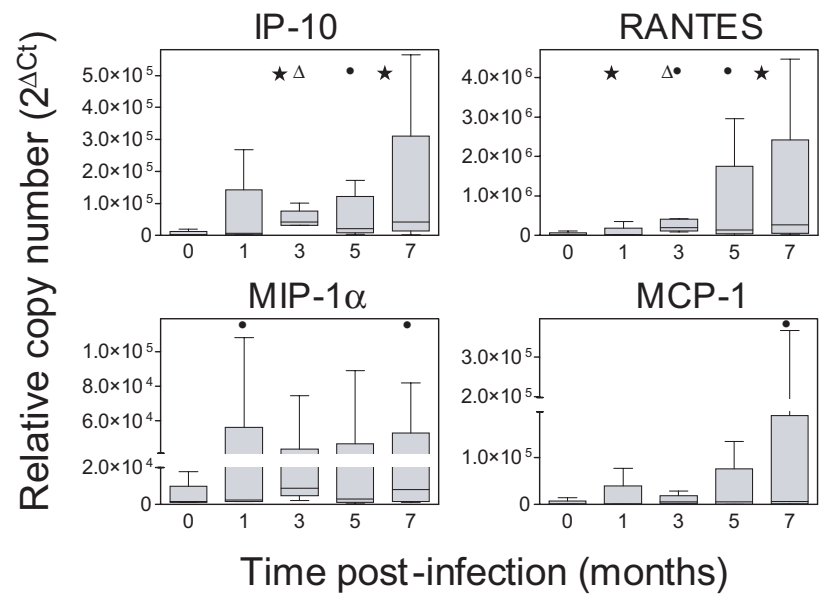

Figure 2. Chemokine gene expression during the course of experimental $L$. infantum infection in dogs. Spleen mRNA expression of IP-10, RANTES, MIP-1 $\alpha$ and MCP-1, analyzed using SYBR green qPCR in EI dogs $(n=6)$. Box plots show the medians (horizontal lines across the box), interquartile ranges (vertical ends of the box), and whiskers (lines extending from the box to the highest and lowest values). Values that are different from pre-infection values $\left(p<0.05^{\star}, p<\right.$ $0.063 \bullet)$ are indicated, as well as those different from uninfected control dogs $(n=2 ; p<0.05 \Delta)$.

post-infection (median fold increase from pre-infection value $(\mathrm{MFI})=64$ and 35 respectively, $p<0.05)$. At 7 months post-infection IFN- $\gamma$ expression still remained high $(\mathrm{MFI}=69 ; p<0.063)$. Differences in the expression of IFN- $\gamma$ were also noted between infected and uninfected dogs at 3 and 5 months postinfection $(p<0.05)$. Interleukin-12 expression did not change markedly during the infection period (Fig. 1), but was higher in infected compared to uninfected dogs at 7 months post-infection $(p<0.05)$. IL-18 (Fig. 1) and TNF- $\alpha$ (data not shown) expression levels remained relatively constant throughout the infection period. The expression level of T-bet, a type-1 associated transcription factor was elevated in infected dogs 3 months post-infection (MFI $=1.7, p<0.05$, Fig. 1).

Cytokines associated with type-2 immune response were also examined. IL-4 expression levels were increased at 1 month post-infection (MFI $=9.1$, $p<0.05$, Fig. 1) and remained elevated at 3 months $(\mathrm{MFI}=6.9, p<0.05)$. At 7 months post-infection IL-4 expression $(\mathrm{MFI}=4)$ had decreased, but was still somewhat elevated compared to the preinfection value $(p<0.063)$. Differences in IL-4 expression compared to uninfected dogs were also noted after 1 month of infection $(p<0.05)$. Interleukin-5 expression at 5 and 7 months post-infection was higher than pre-infection values (MFI values of 5.6 and 6.3, respectively, $p<0.063$, Fig. 1). The expression of GATA-3, a type-2 associated transcription factor remained similar throughout the infection (data not shown). Interestingly, the expression of IL-10 and TGF- $\beta$ did not change during the experimental infection (data not shown).

The expression levels of all chemokines tested in EI dogs increased during the infection period (Fig. 2). More specifically, 

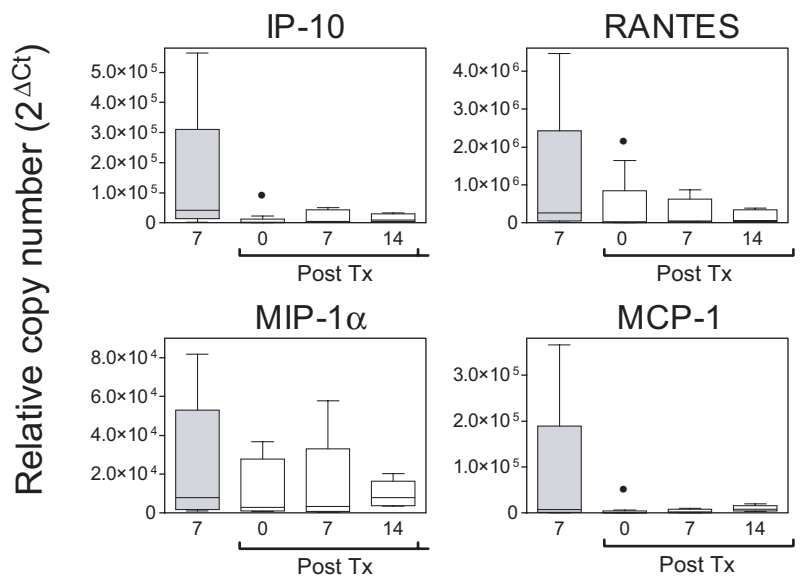

Figure 3. Chemokine gene expression following treatment of experimental $L$. infantum infection. Spleen mRNA expression of IP-10, RANTES, MIP-1 $\alpha$ and MCP-1, was analyzed following treatment of experimental CVL. Box plots show the medians, interquartile ranges, and whiskers as described in the previous figures. Gray box plots indicate values at 7 months post-infection while white box plots show the expression levels at various time points after 3 months of oral allopurinol treatment (post-Tx). Values that are different from pre-treatment values $(p<0.063 \bullet)$ are indicated.

IP-10 expression was elevated in infected dogs compared to their pre-infection values at 3,5 and 7 months post-infection $(\mathrm{MFI}=17.13,9.34$ and 22.7, respectively, $p<0.05, p<0.063$ and $p<0.05$, respectively; Fig. 2). RANTES expression followed a similar pattern (Fig. 2), increasing the above pre-infection values at 1 month post-infection $(\mathrm{MFI}=1.45, p<0.05)$, and remaining elevated at $3,5(\mathrm{MFI}=18.51$ and 13.26, respectively, $p<0.063$ ) and 7 months post-infection $(\mathrm{MFI}=20.19$; $p<0.05)$. Differences in the expression of IP-10 and RANTES were noted between infected and uninfected dogs at 3 months post-infection ( $p<0.05)$. MIP- $1 \alpha$ and MCP-1 expression values in the spleen of EI dogs were elevated $(\mathrm{MFI}=1.8$ and 4.88 for MIP- $1 \alpha$ at 1 and 7 months post-infection, and MFI $=6.82$ for $\mathrm{MCP}-1$ at 7 months post-infection), compared to pre-infection values ( $p<0.063$; Fig. 2). No differences in the expression levels of MIP- $1 \alpha$ and MCP-1 were detected between infected and uninfected dogs.

\subsection{Cytokine, transcription factor and chemokine expression levels in the spleen following treatment}

The expression level of IFN- $\gamma$ dropped noticeably immediately after treatment and was $>13$-fold lower than the level at 7 months post-infection (Tab. II). However, the expression levels of the other cytokines and transcription factors in the spleen of the EI dogs were not different when compared to pre-treatment values (Tab. II).

At the end of treatment (Fig. 3, $t=0$ post-Tx) the expression of IP-10, RANTES and MCP-1 in the spleen of the EI dogs (MFI $=0.73,2.82$, and 0.63, respectively, Fig. 3) was lower than in samples taken just prior to treatment (MFI = 22.7, 20.19, and 6.82, respectively, $p<0.063)$. Interestingly, at 14 months post-treatment MIP- $1 \alpha$ and MCP-1 expression levels were similar to their pretreatment values $(\mathrm{MFI}=3.25$ and 6.92 , respectively), while those of IP-10 and 


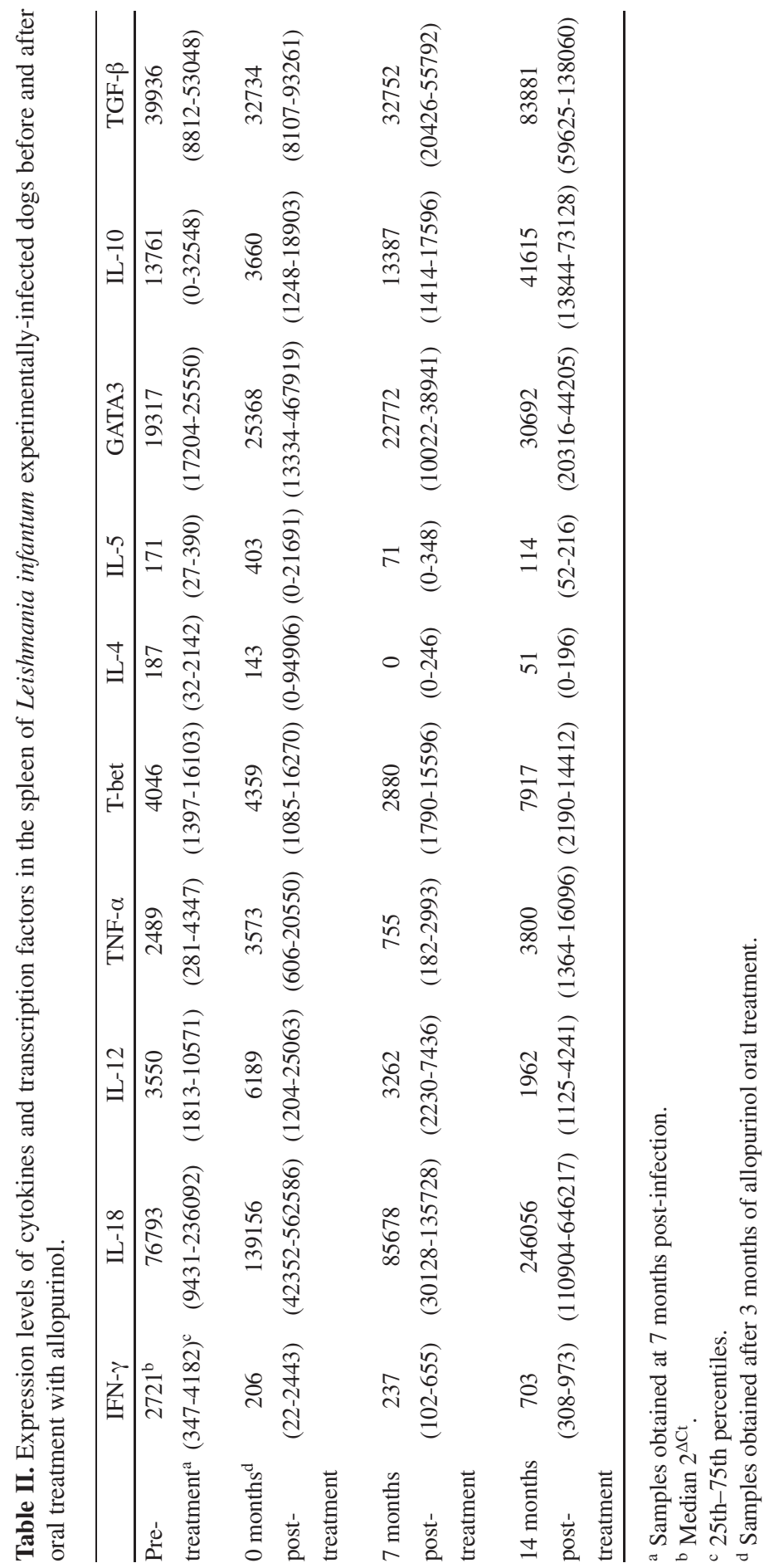


RANTES remained lower $(\mathrm{MFI}=3.19$ and 4.42 , respectively).

\subsection{Cytokine and transcription factor expression levels in the spleen of $L$. infantum naturally-infected dogs}

Cytokine and transcription factor expression levels in the spleen of NI dogs (Tab. III) were similar when compared to values observed for EI dogs at 7 months post-infection (Tab. II, $p>0.1$ for all comparisons). When compared to healthy control dogs (beagle dogs before experimental infection; $n=8$ ) the expression levels of IFN- $\gamma$ and T-bet in the spleens of NI dogs were elevated $(p<0.0001$ and $p<0.05$, respectively, Tab. III). Expression levels of IL-12, IL-18, IL-4 and IL-5 in NI dogs were not higher (Tab. III), and no differences were observed in the expression of TNF- $\alpha$, IL- 10 and TGF- $\beta$ similar to findings with EI dogs (Tabs. II, III).

Chemokine expression levels in the spleens of NI dogs were higher when compared to the control dogs $(p<0.001$ for IP-10 and RANTES; $p<0.05$ for MIP$1 \alpha$ and MCP-1, Fig. 4). Differences were not observed when these levels in NI dogs were compared to EI dogs at 7 months post-infection ( $p>0.4$ for all comparisons).

\subsection{Association between the splenic parasite load and cytokine, transcription factor and chemokine expression levels}

Meaningful statistical correlation between the parasite load in the EI dogs and any of the immunological parameters examined was not observed throughout the post-infection and post-treatment periods. Low rho values were noted for some of the cytokines and chemokines (IL-4, IP-10, TGF- $\beta$ and GATA- $3 ; r=0.3,0.38,-0.37$ and -0.35 respectively, $p<0.05$ ). Interestingly, the fluctuations in the IFN- $\gamma$ MFI levels during the experimental infection were inversely associated with changes observed in the median parasite load. Lower IFN $-\gamma$ MFI was associated with higher parasite load and vice versa. Upon treatment of EI dogs, the expression of IFN- $\gamma$, IP-10, RANTES and MCP-1 decreased considerably, similarly to the splenic parasite load. Statistical correlation between the parasite load and the expression levels of any of the cytokines, transcription factors and chemokines tested in the NI dogs was not found.

\subsection{IgG subclass levels during experimental infection}

A general increase in the amount of IgG and IgG subclass antibodies was observed following experimental infection (Fig. 5). The kinetics of IgG1 and IgG4 increase was similar to that seen for total $\mathrm{IgG}$, plateauing at about 2-3 months and then stabilizing or increasing slowly until 7 months post-infection. On the contrary, $\mathrm{IgG} 2$ and IgG3 levels peaked at 3 months and then decreased over the infection period. Seven months after treatment (Fig. 5), $\mathrm{IgG} 1, \mathrm{IgG} 2$ and $\mathrm{IgG} 3$ were essentially the same as the pre-treatment values, while the amounts of total $\mathrm{IgG}$ and $\mathrm{IgG} 4$ were markedly reduced $(p<0.05)$.

Total IgG, IgG1, IgG2 and IgG4 were positively correlated to IFN- $\gamma$ expression levels during the experimental infection $(r=0.57,0.63,0.62,0.68$ respectively, $p<0.05)$. IgG3 was correlated to a lesser degree with IFN- $\gamma(r=0.48, p<0.05)$. IgG3 and IgG4 also showed poor correlation with IL-18 $(r=0.42, p<0.05)$, and IL-12 ( $r=0.34, p<0.05)$ expression, respectively. Associations were not detected between total IgG and IgG subclasses and the expression levels of other cytokines and transcription factors. 


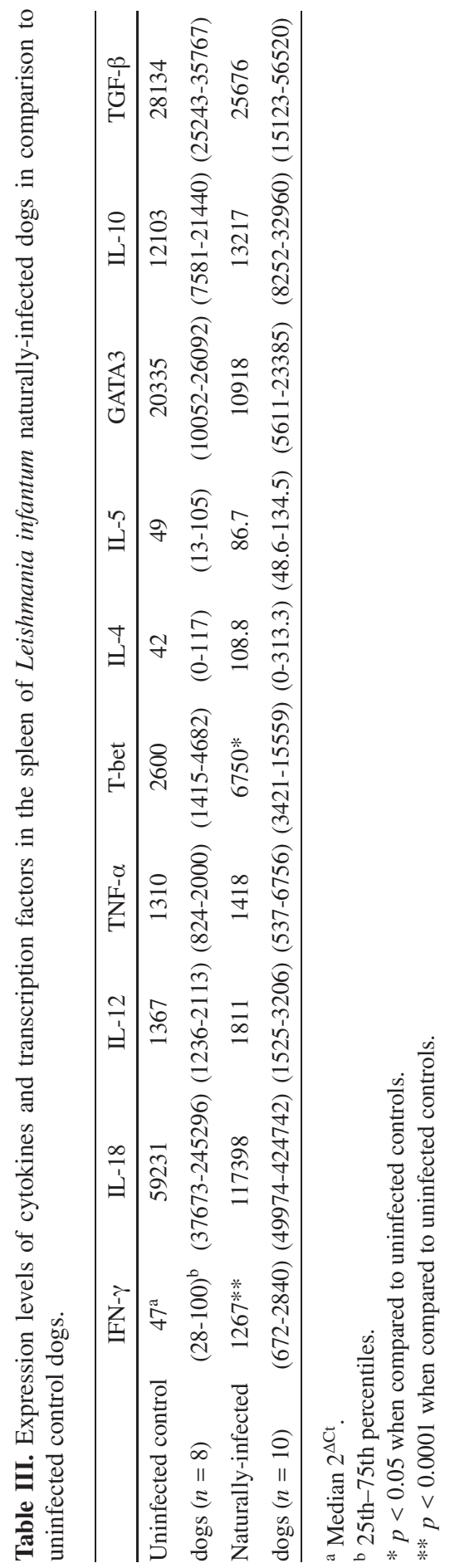




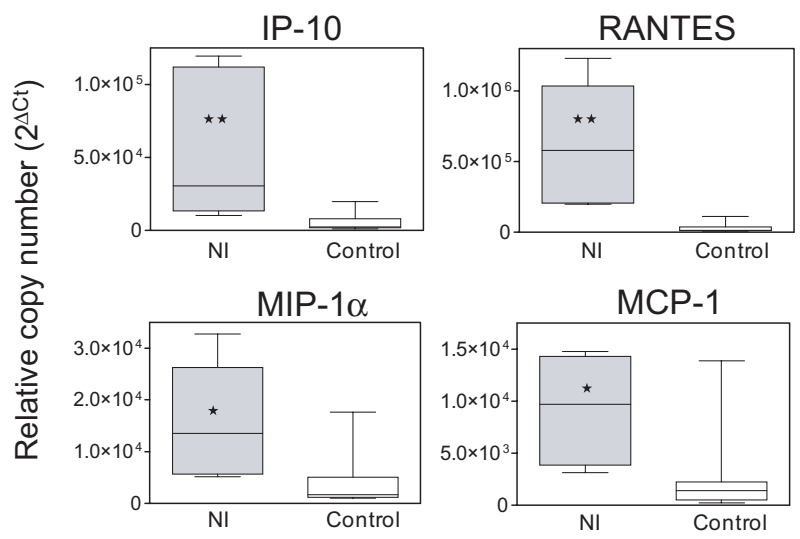

Figure 4. Chemokine gene expression in natural L. infantum infection of dogs. Spleen mRNA expression of IP-10, RANTES, MIP-1 $\alpha$ and MCP-1 in NI dogs $(n=6)$ and control uninfected dogs $(n=8)$. Box plots show the medians, interquartile ranges, and whiskers as described in the previous figures. Gray box plots indicate NI dogs while white box plots indicate control dogs. Values that are different from uninfected control dogs $\left(p<0.0001^{\star \star}\right)$ and $\left(p<0.05^{\star}\right)$ are indicated.

\section{DISCUSSION}

It is now widely accepted that protective immunity against Leishmania parasites is mediated by type- 1 immune responses [26]. This study established that a type-1 immune response is present in the spleen of $L$. infantum-infected dogs. This was indicated by the higher IFN- $\gamma$, T-bet, IP-10, and RANTES mRNA levels found in infected dogs during both oligosymptomatic and polysymptomatic stages of the disease. Type- 2 immune response was manifested as an increase in the expression of IL-4 and IL-5 during oligosymptomatic disease. Our data suggest that during $L$. infantum infection a mixed type- 1 and type- 2 immune response can be found in the spleen of infected dogs. This immune response failed to control the parasites locally.

The two groups of L. infantum-infected dogs included in this study were different in their mode of infection (experimental versus natural), and in the severity of their clinical signs (oligosymptomatic ver- sus polysymptomatic, respectively). However, when the clinical scores for these groups are appraised together with their comparable laboratory results (low albumin to globulin ratio, low hematocrit and low platelet counts), it appears justified to consider them as representatives of different stages in disease development for the purpose of analyzing the immune response.

Fluctuations in the parasite loads during the experimental infection could be inversely associated with IFN- $\gamma$ and IP10 MFI. Although the expression level of IFN- $\gamma$ mRNA in polysymptomatic dogs was higher than uninfected controls, it was still lower than that found in EI oligosymptomatic dogs. This relatively lower IFN- $\gamma$ expression may explain the higher parasite load observed in the spleen of these polysymptomatic dogs. Nevertheless, parasite loads were substantial, even in the presence of high IFN- $\gamma$ expression, implicating that in the spleen of $L$. infantuminfected dogs high levels of IFN- $\gamma$ do not result in the complete elimination of the 

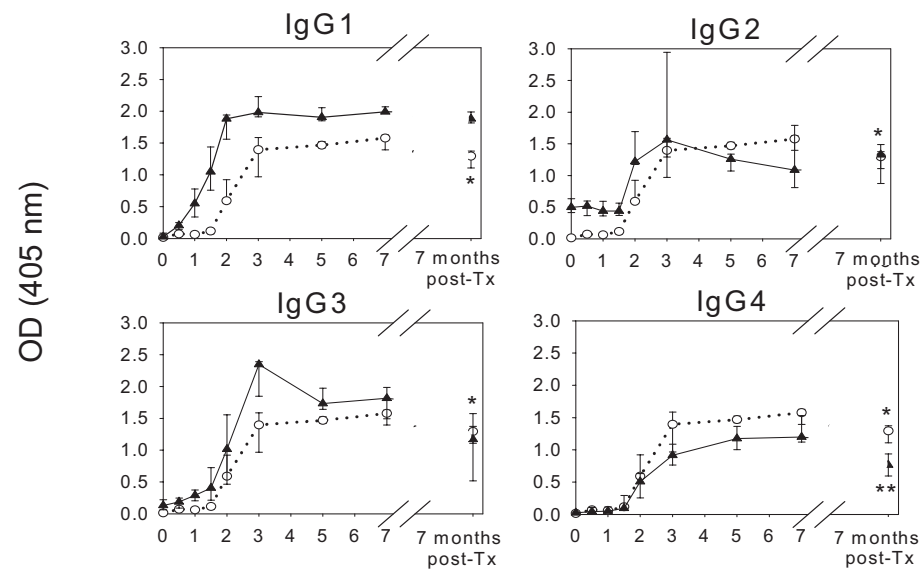

Time post-infection (months)

Figure 5. Parasite specific total $\operatorname{IgG}$ and $\operatorname{IgG}$ subclass values during experimental L. infantum infection and following treatment. Serum titers of Leishmania-specific total IgG (dotted line in each plot) and IgG subclass (IgG1, IgG2, IgG3, IgG4; solid lines) antibodies were evaluated and compared in EI dogs $(n=6)$. Solid triangles (IgG subclasses) and transparent circles (total IgG) represent the median ELISA OD values for all dogs tested. Error bars represent the 25-75th percentiles. The treatment period is indicated by a gap in the plot area. Anti-Leishmania total IgG * and anti-Leishmania IgG4 ${ }^{\star \star}$ were lower 7 months after the treatment was stopped compared to the pre-treatment value $(p<0.05)$.

parasites. One possible explanation is that new parasites are constantly being seeded into the spleen from other highly infected tissues, where IFN- $\gamma$ is not elevated.

High splenic IFN- $\gamma$ was also detected in people infected with $L$. donovani $[19,21,23]$. In contrast to HVL, IL-10 mRNA expression was not elevated in the spleen of $L$. infantum-infected dogs in this study, as was the amount of IL-10 protein in a different study [12]. Interleukin-4, a type-2 immune response cytokine, does not have a clear role in HVL $[19,21]$. Nevertheless, it was significantly elevated relatively early post-infection in the oligosymptomatic EI dogs studied here. Interestingly, even though IL-4 expression was not previously found to be significantly elevated in the bone marrow of L. infantum-infected dogs, higher expression was associated with the presence of more severe clinical signs [31]. Moreover, IL-4 expression in skin biopsies obtained from infected dogs was found to be significantly higher than in similar samples taken from uninfected dogs and was correlated with increased parasite load in skin lesions [7]. We hypothesize that the suppressive effect of IL-4 on macrophage activation [4] during the early stages of the infection could have facilitated parasite survival and replication, and the inability of a host type-1 immune response to eliminate the parasites.

The most noticeable immunological change in the dogs' response to treatment were the decreases in the expression levels of IFN- $\gamma$ (13.2 fold lower), IP-10 (31.2 fold lower), RANTES (7.2 fold lower) and MCP-1 (10.8 fold lower) when compared to pre-treatment values. Similarly, parasite load in the spleens of the oligosymptomatic dogs was high during the infection but decreased after treatment $(t=0,1.3 \times$ $10^{4}$ fold lower, $p<0.05$ ). As demonstrated by the minimal number of parasites and 
lack of clinical signs during the 14 months follow-up, clinical response of the EI dogs to this treatment regimen in the oligosymptomatic stage of the disease was good. We assume that the decrease we observed in chemokine and IFN- $\gamma$ mRNA levels reflects the reduced recruitment of immune cells into the spleen following treatment, due to the minimal amount of parasites remaining in the tissue. This assumption can also be corroborated by the fact that the spleen was not enlarged after treatment.

To our knowledge, this is the first study to examine chemokine expression in the spleen of a natural host with visceral leishmaniasis. Experimental and natural infection of dogs with L. infantum caused an up-regulation of the chemokines tested in the spleen, although in the oligosypmtomatic dogs, only IP-10 and RANTES were markedly elevated. The elevated levels of the tested chemokines in this study are suggestive of an accumulation of infiltrating monocytes attracted by MIP1- $\alpha$ and MCP-1, as well as of CD4+Th1 and CD8+ cells which could have been recruited by IP-10 and induced further IP-10 expression through IFN- $\gamma$ secretion. This is true also for later stages of the disease ( 7 months post-experimental infection and in natural infection). Not surprisingly, the expression level of IP-10 was relatively lower in NI when compared to EI dogs, while those of RANTES, MIP- $1 \alpha$ and MCP-1 were higher in the NI dogs. The higher IP-10 expression in EI dogs is consistent with the higher IFN- $\gamma$ expression observed in these dogs and with IP-10 being induced by IFN- $\gamma$.

It has been previously described in mice that the production of IgG1 is associated with the Th2 type response and that of IgG2a is associated with the Th1 type response [39]. Earlier studies to determine if specific IgG subclass levels in L. infantum-infected dogs are associated with the clinical outcome and hence with resistance or susceptibility to the disease, produced conflicting results $[6,14,24,38]$. However, these studies used commercial polyclonal anti-sera to canine $\operatorname{IgG} 1$ and $\mathrm{IgG} 2$, while we used the same monoclonal antibody panel (IgG1, IgG2, IgG3 and IgG4) previously employed by Quinnell et al. [31,32]. We found a general up-regulation of the total $\mathrm{IgG}$, as well as of all $\mathrm{IgG}$ subclasses during experimental infection similarly to what was previously found for L. infantum NI dogs using this antibody panel, and also for HVL patients $[11,32]$. This finding, together with the inability to associate specific cytokine patterns with specific IgG subclasses during the course of the experimental infection and following the treatment, further implies that specific canine IgG subclasses cannot be directly associated with resistance or susceptibility to CVL, as was previously suggested $[14,32]$.

This study established that a type- 1 immune response was the dominant response in the spleen during visceral leishmaniasis in the dog. Moreover, the expression of various chemokines with roles in $\mathrm{T}$ cell activation, differentiation, recruitment and other effector mechanisms was found to be elevated in the spleen of $L$. infantuminfected dogs. Elevated IL-4 mRNA was noted early during infection and is thought to have a role in the persistence of parasites in the spleen in the presence of high IFN- $\gamma$, IP-10, and RANTES expression. Future studies are needed in order to clarify the association between the trafficking of various cell populations, cytokine and chemokine gradients, and the presence of parasites in the spleen, as well as the association with the immune responses and parasite loads in other visceral and peripheral organs. The differences that we observed between the splenic immune response in dogs and previous studies in humans further suggest that the nature of the immune response to infection with Leishmania parasites is not solely dependent on the specific Leishmania sp. involved, but has a considerable host-dependent component. 


\section{ACKNOWLEDGEMENTS}

The authors thank Dr Liat Gonen for her help, Dr Michael J. Day for the canine IgG subclass MAbs, and Dr Reinhard K. Straubinger for sharing the sequence of the canine IL-10 primers. This work was supported by the Center for the Study of Emerging Diseases, Yeshaya Horowitz Foundation. The corresponding author was supported by a fellowship from the Yeshaya Horowitz Foundation.

\section{REFERENCES}

[1] Bacon K.B., Premack B.A., Gardner P., Schall T.J., Activation of dual T cell signaling pathways by the chemokine RANTES, Science (1995) 269:1727-1730.

[2] Baneth G., Dank G., Keren-Kornblatt E., Sekeles E., Adini I., Eisenberger C.L., Schnur L.F., King R., Jaffe C.L., Emergence of visceral leishmaniasis in central Israel, Am. J. Trop. Med. Hyg. (1998) 59:722-725.

[3] Belkaid Y., Hoffmann K.F., Mendez S., Kamhawi S., Udey M.C., Wynn T.A., Sacks D.L., The role of interleukin (IL)-10 in the persistence of Leishmania major in the skin after healing and the therapeutic potential of anti-IL-10 receptor antibody for sterile cure, J. Exp. Med. (2001) 194:1497-1506.

[4] Bogdan C., Vodovotz Y., Paik J., Xie Q.W., Nathan C., Mechanism of suppression of nitric oxide synthase expression by interleukin-4 in primary mouse macrophages, J. Leukoc. Biol. (1994) 55:227-233.

[5] Borja-Cabrera G.P., Correia Pontes N.N., da Silva V.O., Paraguai de Souza E., Santos W.R., Gomes E.M., Luz K.G., Palatnik M., Palatnik de Sousa C.B., Long lasting protection against canine kala-azar using the FML-QuilA saponin vaccine in an endemic area of Brazil (Sao Goncalo do Amarante, RN), Vaccine (2002) 20:3277-3284.

[6] Bourdoiseau G., Bonnefont C., Hoareau E., Boehringer C., Stolle T., Chabanne L., Specific IgG1 and IgG2 antibody and lymphocyte subset levels in naturally Leishmania infantum-infected treated and untreated dogs, Vet. Immunol. Immunopathol. (1997) 59:21-30.

[7] Brachelente C., Muller N., Doherr M.G., Sattler U., Welle M., Cutaneous leishmaniasis in naturally infected dogs is associated with a $\mathrm{T}$ helper-2-biased immune response, Vet. Pathol. (2005) 42:166-175.

[8] Brandonisio O., Panaro M.A., Fumarola I., Sisto M., Leogrande D., Acquafredda A., Spinelli R., Mitolo V., Macrophage chemotactic protein-1 and macrophage inflammatory protein-1 alpha induce nitric oxide release and enhance parasite killing in Leishmania infantum-infected human macrophages, Clin. Exp. Med. (2002) 2:125-129.

[9] Brenier-Pinchart M.P., Pelloux H., DerouichGuergour D., Ambroise-Thomas P., Chemokines in host-protozoan-parasite interactions, Trends Parasitol. (2001) 17:292-296.

[10] Chamizo C., Moreno J., Alvar J., Semiquantitative analysis of cytokine expression in asymptomatic canine leishmaniasis, Vet. Immunol. Immunopathol. (2005) 103:67-75.

[11] Chatterjee M., Basu K., Basu D., Bannerjee D., Pramanik N., Guha S.K., Goswami R.P., Saha S.K., Mandal C., Distribution of IgG subclasses in antimonial unresponsive Indian kala- azar patients, Clin. Exp. Immunol. (1998) 114:408-413.

[12] Correa A.P., Dossi A.C., de Oliveira Vasconcelos R., Munari D.P., de Lima V.M., Evaluation of transformation growth factor beta(1), interleukin-10, and interferon-gamma in male symptomatic and asymptomatic dogs naturally infected by Leishmania (Leishmania) chagasi, Vet. Parasitol. (2006) 143:267-274.

[13] Cotterell S.E., Engwerda C.R., Kaye P.M., Leishmania donovani infection initiates $\mathrm{T}$ cell-independent chemokine responses, which are subsequently amplified in a $\mathrm{T}$ cell-dependent manner, Eur. J. Immunol. (1999) 29:203-214.

[14] Deplazes P., Smith N.C., Arnold P., Lutz H., Eckert J., Specific IgG1 and IgG2 antibody responses of dogs to Leishmania infantum and other parasites, Parasite Immunol. (1995) 17:451-458.

[15] Dorner B.G., Scheffold A., Rolph M.S., Huser M.B., Kaufmann S.H., Radbruch A., Flesch I.E., Kroczek R.A., MIP1alpha, MIP-1beta, RANTES, and ATAC/lymphotactin function together with IFN-gamma as type 1 cytokines, Proc. Natl. Acad. Sci. USA (2002) 99:6181-6186.

[16] Dufour J.H., Dziejman M., Liu M.T., Leung J.H., Lane T.E., Luster A.D., IFN-gammainducible protein 10 (IP-10; CXCL10)- 
deficient mice reveal a role for IP-10 in effector $\mathrm{T}$ cell generation and trafficking, $\mathrm{J}$. Immunol. (2002) 168:3195-3204.

[17] Frangogiannis N.G., Mendoza L.H., Smith C.W., Michael L.H., Entman M.L., Induction of the synthesis of the $\mathrm{C}-\mathrm{X}-\mathrm{C}$ chemokine interferon-gamma-inducible protein-10 in experimental canine endotoxemia, Cell Tissue Res. (2000) 302:365-376.

[18] Gavagani A.S.M., Mohite H., Edrissian G.H., Mohebali M., Davies C.R., Domestic dog ownership in Iran is a risk factor for human infection with Leishmania infantum, Am. J. Trop. Med. Hyg. (2002) 67:511-515.

[19] Ghalib H.W., Piuvezam M.R., Skeiky Y.A., Siddig M., Hashim F.A., el Hassan A.M., Russo D.M., Reed S.G., Interleukin 10 production correlates with pathology in human Leishmania donovani infections, J. Clin. Invest. (1993) 92:324-329.

[20] Hailu A., van der Poll T., Berhe N., Kager P.A., Elevated plasma levels of interferon (IFN)-gamma, IFN-gamma inducing cytokines, and IFN-gamma inducible CXC chemokines in visceral leishmaniasis, Am. J. Trop. Med. Hyg. (2004) 71:561-567.

[21] Karp C.L., el Safi S.H., Wynn T.A., Satti M.M., Kordofani A.M., Hashim F.A., HagAli M., Neva F.A., Nutman T.B., Sacks D.L., In vivo cytokine profiles in patients with kala-azar. Marked elevation of both interleukin-10 and interferon-gamma, J. Clin. Invest. (1993) 91:1644-1648.

[22] Karpus W.J., Lukacs N.W., Kennedy K.J., Smith W.S., Hurst S.D., Barrett T.A., Differential CC chemokineinduced enhancement of $\mathrm{T}$ helper cell cytokine production, J. Immunol. (1997) 158:4129-4136.

[23] Kenney R.T., Sacks D.L., Gam A.A., Murray H.W., Sundar S., Splenic cytokine responses in Indian kala-azar before and after treatment, J. Infect. Dis. (1998) 177:815-818.

[24] Leandro C., Santos-Gomes G.M., Campino L., Romao P., Cortes S., Rolao N., GomesPereira S., Rica Capela M.J., Abranches P., Cell mediated immunity and specific IgG1 and $\mathrm{IgG} 2$ antibody response in natural and experimental canine leishmaniosis, Vet. Immunol. Immunopathol. (2001) 79:273-284

[25] Mazza G., Whiting A.H., Day M.J., Duffus W.P., Development of an enzyme-linked immunosorbent assay for the detection of IgG subclasses in the serum of normal and diseased dogs, Res. Vet. Sci. (1994) 57:133-139.

[26] McMahon-Pratt D., Alexander J., Does the Leishmania major paradigm of pathogenesis and protection hold for New World cutaneous leishmaniases or the visceral disease? Immunol. Rev. (2004) 201:206-224.

[27] Miralles G.D., Stoeckle M.Y., McDermott D.F., Finkelman F.D., Murray H.W., Th1 and Th2 cell-associated cytokines in experimental visceral leishmaniasis, Infect. Immun. (1994) 62:1058-1063.

[28] Noli C., Auxilia S.T., Treatment of canine Old World visceral leishmaniasis: a systematic review, Vet. Dermatol. (2005) 16:213-232.

[29] Peters I.R., Helps C.R., Hall E.J., Day M.J., Real-time RT-PCR: considerations for efficient and sensitive assay design, J. Immunol. Methods (2004) 286:203-217.

[30] Peters I.R., Helps C.R., Calvert E.L., Hall E.J., Day M.J., Cytokine mRNA quantification in histologically normal canine duodenal mucosa by real-time RTPCR, Vet. Immunol. Immunopathol. (2005) 103:101-111.

[31] Quinnell R.J., Courtenay O., Shaw M.A., Day M.J., Garcez L.M., Dye C., Kaye P.M., Tissue cytokine responses in canine visceral leishmaniasis, J. Infect. Dis. (2001) 183:1421-1424.

[32] Quinnell R.J., Courtenay O., Garcez L.M., Kaye P.M., Shaw M.A., Dye C., Day M.J., IgG subclass responses in a longitudinal study of canine visceral leishmaniasis, Vet. Immunol. Immunopathol. (2003) 91:161-168.

[33] Rabin R.L., Park M.K., Liao F., Swofford R., Stephany D., Farber J.M., Chemokine receptor responses on $\mathrm{T}$ cells are achieved through regulation of both receptor expression and signaling, J. Immunol. (1999) 162:3840-3850.

[34] Ritter U., Moll H., Laskay T., Brocker E., Velazco O., Becker I., Gillitzer R., Differential expression of chemokines in patients with localized and diffuse cutaneous American leishmaniasis, J. Infect. Dis. (1996) 173:699-709.

[35] Rousseau D., Demartino S., Anjuere F., Ferrua B., Fragaki K., Le Fichoux Y., Kubar J., Sustained parasite burden in the spleen of Leishmania infantum-infected $\mathrm{BALB} / \mathrm{c}$ mice is accompanied by expression of MCP-1 transcripts and lack of protec- 
tion against challenge, Eur. Cytokine Netw. (2001) 12:340-347.

[36] Santos-Gomes G.M., Rosa R., Leandro C., Cortes S., Romao P., Silveira H., Cytokine expression during the outcome of canine experimental infection by Leishmania infantum, Vet. Immunol. Immunopathol. (2002) 88:21-30.

[37] Scott P., Natovitz P., Coffman R.L., Pearce E., Sher A., Immunoregulation of cutaneous leishmaniasis. T cell lines that transfer protective immunity or exacerbation belong to different $\mathrm{T}$ helper subsets and respond to distinct parasite antigens, J. Exp. Med. (1988) 168:1675-1684.

[38] Solano-Gallego L., Riera C., Roura X., Iniesta L., Gallego M., Valldares J.E., Fisa R., Castillejo S., Alberola J., Ferrer L., Arboix M., Portus M., Leishmania infantum-specific IgG, IgG1 and $\mathrm{IgG} 2$ antibody responses in healthy and ill dogs from endemic areas - Evolution in the course of infection and after treatment, Vet. Parasitol. (2001) 96:265-276.

[39] Stevens T.L., Bossie A., Sanders V.M., Fernandez-Botran R., Coffman R.L., Mosmann T.R., Vitetta E.S., Regulation of antibody isotype secretion by subsets of antigen-specific helper T cells, Nature (1988) 334:255-258.
[40] Strauss-Ayali D., Jaffe C.L., Burshtain O., Gonen L., Baneth G., Polymerase chain reaction using noninvasively obtained samples, for the detection of Leishmania infantum DNA in dogs, J. Infect. Dis. (2004) 189:1729-1733.

[41] Strauss-Ayali D., Baneth G., Shor S., Okano F., Jaffe C.L., Interleukin-12 augments a Th1-type immune response manifested as lymphocyte proliferation and interferon gamma production in Leishmania infantum-infected dogs, Int. J. Parasitol. (2005) 35:63-73.

[42] Teixeira M.J., Teixeira C.R., Andrade B.B., Barral-Netto M., Barral A., Chemokines in host-parasite interactions in leishmaniasis, Trends Parasitol. (2005) 22:32-40.

[43] Wortmann G., Sweeney C., Houng H.S., Aronson N., Stiteler J., Jackson J., Ockenhouse C., Rapid diagnosis of leishmaniasis by fluorogenic polymerase chain reaction, Am. J. Trop. Med. Hyg. (2001) 65:583-587.

[44] Zaph C., Scott P., Interleukin-12 regulates chemokine gene expression during the early immune response to Leishmania major, Infect. Immun. (2003) 71:1587-1589. 